\title{
Clinical Validation and Applicability of Different Tipranavir/ritonavir Genotypic Scores in HIV-1 Protease Inhibitor-Experienced Patients
}

\author{
Annalisa Saracino, ${ }^{*}$, Laura Monno ${ }^{2}$, Alessandra Tartaglia ${ }^{1}$, Carmine Tinelli $^{3}$, Elena Seminari ${ }^{3}$, \\ Franco Maggiolo ${ }^{4}$, Stefano Bonora ${ }^{5}$, Stefano Rusconi ${ }^{6}$, Valeria Micheli ${ }^{7}$, Sergio Lo Caputo ${ }^{8}$, \\ Laura Lazzaroni ${ }^{2}$, Sergio Ferrara ${ }^{1}$, Nicoletta Ladisa ${ }^{2}$, Paola Nasta ${ }^{10}$, Giustino Parruti ${ }^{11}$, \\ Rita Bellagamba ${ }^{12}$, Federica Forbici ${ }^{12}$ and Gioacchino Angarano ${ }^{1}$
}

\begin{abstract}
${ }^{I}$ Clinic of Infectious Diseases, University of Foggia; ${ }^{2}$ Clinic of Infectious Diseases, University of Bari; ${ }^{3}$ Clinical Epidemiology and biometric Unit, Fondazione IRCCS S. Matteo, Pavia; ${ }^{4}$ Division of Infectious Diseases, Ospedali Riuniti, Bergamo; ${ }^{5}$ Department of Infectious Diseases, University of Turin; ${ }^{6}$ Institute of Infectious and Tropical Diseases, L. Sacco Hospital, University of Milan; ${ }^{7}$ II Division of Infectious Diseases, L. Sacco Hospital, Milan; ${ }^{8}$ Division of Infectious Diseases, S M. Annunziata Hospital, Florence; ${ }^{9}$ Department of Infectious Diseases, University of Insubria, Varese; ${ }^{10}$ Institute of Infectious and Tropical Diseases, University of Brescia; ${ }^{11}$ Unit of Infectious Diseases, Ospedale Civile Spirito Santo, Pescara; ${ }^{12}$ National Institute for Infectious Diseases IRCCS Lazzaro Spallanzani, Rome, Italy
\end{abstract}

\begin{abstract}
Tipranavir, a non-peptidic protease inhibitor which shows in vitro efficacy against some HIV-1-resistant strains, can be used in salvage therapies for multi-experienced HIV patients due to its peculiar resistance profile including 21 mutations at 16 protease positions according to International AIDS Society (IAS). Other genotypic scores, however, which attribute a different weight to single amino-acid substitutions, have been recently proposed. To validate the clinical utility of four different genotypic scores for selecting tipranavir responders, the baseline resistance pattern of $176 \mathrm{HIV}$ heavily experienced patients was correlated with virological success (HIV-RNA $<50$ copies/ml) after 24 weeks of a new treatment based on tipranavir/ritonavir. Virological suppression after 24 weeks was reached by $42.5 \%$ of patients. With univariate analysis, genotypic scores were all associated with outcome but showed a low accuracy with ROC analysis, with the weighted score (WS) by Scherer et al. demonstrating the best performance with an AUC of $68 \%$. Only 52\% of patients classified as susceptible $(\mathrm{WS} \leq 3)$ responded to the new therapy. The following variables were significantly associated $(\mathrm{p}<0.05)$ to failure with multivariate analysis: WS, log peak of HIV-RNA, IAS mutations: L33F, I54AMV, Q58E, and non-IAS mutation: N37DES. On the contrary, the use of T20 in T20-naïve patients and the V82AFSI and F53LY non-IAS mutations were associated with virological success. The study suggests that even if the "weighted" scores are able to interpret correctly the antiretroviral resistance profile of multi-experienced patients, it is difficult to individuate a cut-off which can be easily applied to this population for discriminating responders.
\end{abstract}

Keywords: Antiretrovirals, protease inhibitors, genotypic resistance, tipranavir.

\section{INTRODUCTION}

In the HAART era, a wide antiretroviral resistance, particularly if extended to all drug classes, is still associated with a poor clinical outcome and represents a risk-marker for disease progression and mortality [1]. However, the results of recent trials with new boosted protease inhibitors (PIs), such as tipranavir/ritonavir (TPV/r) [2-4] and darunavir/ ritonavir [5] which demonstrate efficacy as salvage therapy in a percentage of patients ranging from $20 \%$ to $60 \%$, have permitted specialists to update the guidelines for treatment of highly antiretroviral-experienced HIV-infected patients [6]. Virological suppression is now considered possible also for multi-experienced patients when treated with at least two active drugs from different classes, eventually including entry inhibitors, integrase inhibitors and, in the near future, other drugs in advanced stages of development.

\footnotetext{
*Address correspondence to this author at the Clinic of Infectious Diseases, University of Foggia, v.le L. Pinto, $1-71100$ Foggia, Tel/Fax: +39 0881 732215; E-mail: a.saracino@medicina.unifg.it
}

The results of these phase II/III clinical trials, nevertheless, should always be evaluated in the context of clinical practice with regard to drug effectiveness and predictive power for genotypic resistance patterns, thereby avoiding a selection bias due to inherent systematic differences between the trial population and the general patient population. This is particularly true for tipranavir, a PI which shows in vitro activity against some PI-resistant HIV-1 strains [7, 8], and whose resistance profile seems to require multiple protease mutations to determine a reduced susceptibility. In fact, while four main mutations in the protease gene (at positions 33, 82, 84 and 90) were originally associated with TPV/r resistance, a more recent large database analysis has revealed that at least 21 mutations at 16 positions in the protease gene (L10V, I13V, K20M/R/V, L33F, E35G, M36I, K43T, M46L, I47V, I54A/M/V, Q58E, $\mathrm{H} 69 \mathrm{~K}, \mathrm{~T} 74 \mathrm{P}, \mathrm{V} 82 \mathrm{~L} / \mathrm{T}, \mathrm{N} 83 \mathrm{D}$, and $\mathrm{I} 84 \mathrm{~V}$ ) are involved in determining TPV/r loss of susceptibility, some of which are not associated with resistance to any other PIs $[9,10]$. Some perplexities regarding the validity of the current $\mathrm{TPV} / \mathrm{r}$ score 
(TS) [9] have been advanced in recent reports [11] ${ }^{1,2}$ which underlined the importance of attributing a different weight to diverse amino acid variants for each protease positions, with some substitutions improving rather than reducing the response to TPV/r. In an initial report by Parkin et al. ${ }^{1}$, the relationship between genotypic TPV/r resistance-associated mutations and the fold-change in phenotypic susceptibility to the drug in clinical isolates was analyzed and several additional mutations were identified as associated with fold change either significantly higher or lower than expected; therefore, a revised TPV/r score (PS) assigning a variable weighting to different mutations has been proposed. When studying the correlation between genotype and virological response in a multi PI-experienced patient cohort, Marcelin et al. [11] also suggested a different score (MS) associating some mutations with failure, and others $(53 \mathrm{~L} / \mathrm{W} / \mathrm{Y})$ with response to $\mathrm{TPV} / \mathrm{r}$.

Lastly, a TPV/r-weighted score (WS) ${ }^{2}$ has been recently developed using data from the RESIST trials in which only mutations I47V, I54AVM, Q58E, T74P, V82LT, and N83D showed the greatest weights in influencing virological failure, while other mutations (L24I, I50LV, I54L, L76V), not included in the current TPV/r score, were associated to an increased TPV/r susceptibility.

In the present study, the protease resistance pattern at baseline was correlated with virological success (HIVRNA $<50$ copies $/ \mathrm{ml}$ ) after 24 weeks of treatment in a population of $176 \mathrm{HIV}$ patients enrolled in the Italian Expanded Access Program for Tipranavir, with the aim of evaluating the clinical applicabiliy of the above mentioned $\mathrm{TPV} / \mathrm{r}$ genotypic scores for discriminating possible TPV/r responders.

\section{PATIENTS AND METHODS}

\section{Study Population}

A total of 176 HIV-1 infected patients who were enrolled in the Italian Early Access Program (EAP BI 1182.16 study) in the period between November 2004 and January 2006 were administered a tipranavir/ritonavir (TPV/r)-based regimen. The study was reviewed and approved by the Independent Ethics Committee of each participating Center. Only patients for whom a baseline genotypic resistance profile was available were included in the analysis. According to the TPV/r EAP inclusion criteria, patients were $>18$-years old, previously treated with all three antiretroviral classes including at least two PI-based regimens, and failing or intolerant to other approved antiretroviral treatments. The use of other investigational drugs within 30 days prior to $\mathrm{TPV} / \mathrm{r}$ initiation and baseline hepatic impairment (AST and/or ALT values more than three times the upper normal limit) represented a criteria for exclusion. All patients provided a written informed consent.

\footnotetext{
${ }^{1}$ Parkin L, Chappey C. Protease mutations associated with a higher or lower than expected tipranavir (TPV) susceptibility based on the TPV mutation score. Presented at: XIII Conference on Retrovirus and Opportunistic Infections; 5-8 February 2006; Denver, CO, USA. Abstract 637.

${ }^{2}$ Scherer J, Boucher CA, Baxter JD, et al. Improving the prediction of virologic response to tipranavir: the development of a tipranavir weighted score. Presented at: at: XI European AIDS Conference Society; 24 - 27 October 2007; Madrid, Spain. Poster P3.4/07.
}

\section{Methods}

CD4+ cell counts were performed locally using standard flow cytometry. Plasma HIV-1 RNA was assessed in each centre participating in study by means of branched DNA (Versant RNA, version 3.0 [Bayer]), Amplicor (Monitor test, version 1.5 [Roche Diagnostics]) or nucleic acid sequencebased amplification (Nuclisens, version 2.1 [Bio-Merieux]); the highest limit of HIV-RNA detection accepted was 50 copies.

Baseline HIV-1 genotyping test was also performed locally using either the 6.0 and 7.0 TrueGene kit (Bayer) or the Viro-Seq kit (Abbott). The optimized background regimen (OBR) was selected by the investigators based on genotypic data. The Sequence Analysis Program of the Stanford HIV RT and Protease Sequence Database (http://hivdb.stanford.edu/hiv/) (version 4.1.9) was used for the interpretation of mutational profiles. No resistance testing was performed for T-20, which was included in the new regimen based on patient clinical history and physician judgement.

HIV-1 pol sequences (GenBank accession numbers EU007912-EU008077) were then retrospectively collected by the Coordinating Center and compared to a reference sequence of HIV-1 subtype B with the use of the free online Sequence Analysis Program (http://hivdb.Stanford.edu) which also provided a subtype assignment; genotypic data were reported as amino acid substitutions with respect to the consensus B. The substitutions at positions associated with $\mathrm{TPV} / \mathrm{r}$ drug-resistance according to the IAS-USA list [10] were all included in the analysis; the amino acid variants other than those indicated by IAS were analyzed separately. For the additional positions within the protease domain, all the amino acid variants differing from the consensus were considered as mutations, but only those detected in at least $10 \%$ of patients were included in the analysis.

Four different genotypic scores: original TPV/r score (TS) [9], Scherer score (WS) ${ }^{2}$, Parkin score (PS) ${ }^{1}$ and Marcelin score (MS) [11] were calculated.

For TS [9], one point was assigned for the presence of each of the following mutations: $\mathrm{L} 10 \mathrm{~V}, \mathrm{I} 13 \mathrm{~V}, \mathrm{~K} 20 \mathrm{M} / \mathrm{R} / \mathrm{V}$, L33F, E35G, M36I, K43T, M46L, I47V, I54A/M/V, Q58E, $\mathrm{H} 69 \mathrm{~K}, \mathrm{~T} 74 \mathrm{P}, \mathrm{V} 82 \mathrm{~L} / \mathrm{T}, \mathrm{N} 83 \mathrm{D}$, and I84V (score range: 0 to $+16)$.

For $\mathrm{WS}^{2}$, the score was calculated as follows: $\mathrm{L} 10 \mathrm{~V}=1$, $\mathrm{I} 13 \mathrm{~V}=0, \mathrm{~K} 20 \mathrm{M} / \mathrm{R} / \mathrm{V}=0, \mathrm{~L} 33 \mathrm{~F}=-2, \mathrm{E} 35 \mathrm{G}=0, \mathrm{M} 36 \mathrm{I}=2$, $\mathrm{K} 43 \mathrm{~T}=2, \mathrm{M} 46 \mathrm{~L}=1, \mathrm{I} 47 \mathrm{~V}=6, \mathrm{I} 50 \mathrm{LV}=-4, \mathrm{I} 54 \mathrm{~A} / \mathrm{M} / \mathrm{V}=3$, $\mathrm{I} 54 \mathrm{~L}=-7, \mathrm{Q} 58 \mathrm{E}=5, \mathrm{H} 69 \mathrm{~K}=0, \mathrm{~T} 74 \mathrm{P}=6, \mathrm{~V} 82 \mathrm{~L} / \mathrm{T}=5, \mathrm{~N} 83 \mathrm{D}=$ 4 , and $\mathrm{I} 84 \mathrm{~V}=2$ (score range: -13 to +37 ).

For PS ${ }^{1}$, the following score system was applied: $\mathrm{L} 10 \mathrm{VI}=0.5, \mathrm{~V} 11 \mathrm{~L}=1, \mathrm{I} 13 \mathrm{~V}=0.5, \mathrm{~K} 20 \mathrm{M} / \mathrm{V}=1, \mathrm{~K} 20 \mathrm{R}=0.5$, $\mathrm{L} 24 \mathrm{I}=-1, \mathrm{D} 30 \mathrm{~N}=-1, \mathrm{~V} 32 \mathrm{I}=1, \mathrm{~L} 33 \mathrm{~F}=1, \mathrm{E} 35 \mathrm{G}=1, \mathrm{M} 36 \mathrm{I}=1$, $\mathrm{K} 43 \mathrm{~T}=1, \mathrm{M} 46 \mathrm{IL}=0.5, \mathrm{I} 47 \mathrm{~V}=2, \mathrm{I} 50 \mathrm{LV}=-1, \mathrm{I} 54 \mathrm{M} / \mathrm{V}=1$, $\mathrm{I} 54 \mathrm{~L}=-1, \mathrm{Q} 58 \mathrm{E}=1, \mathrm{H} 69 \mathrm{~K}=1, \mathrm{~A} 71 \mathrm{~L}=1, \mathrm{G} 73 \mathrm{~T}=1, \mathrm{~T} 74 \mathrm{P}=1$, $\mathrm{L} 76 \mathrm{~V}=-1, \mathrm{~V} 82 \mathrm{I}=-1, \mathrm{~V} 82 \mathrm{~L}=1, \mathrm{~V} 82 \mathrm{~T}=2, \mathrm{~N} 83 \mathrm{D}=1, \mathrm{I} 84 \mathrm{~V}=$ $2, \mathrm{~L} 89 \mathrm{~V}=1$, and $\mathrm{L} 90 \mathrm{M}=0.5$ (score range : -6 to +24.5 ).

Finally, for MS [11] one point was assigned to mutations: $36 \mathrm{I} / \mathrm{L} / \mathrm{V}, 58 \mathrm{E}, 69 \mathrm{I} / \mathrm{K} / \mathrm{N} / \mathrm{Q} / \mathrm{R} / \mathrm{Y}$ and $89 \mathrm{I} / \mathrm{M} / \mathrm{R} / \mathrm{T} / \mathrm{V}$, while a -1 value was attributed to the $53 \mathrm{~L} / \mathrm{W} / \mathrm{Y}$ mutation (score range: 1 to +4$)$. 
Antiretroviral drug resistance was also evaluated using the Virtual Phenotype ${ }^{\mathrm{TM}}$-LM (Virco) (VP). At the time of analysis, the clinical cut-off values for tipranavir were based on treatment response in patients enrolled in the RESIST trials [2-4]; a maximum response was foreseen for isolates with a predicted fold-change in $\mathrm{IC}_{50}$ below the lower clinical cut-off $(=1.2)$, a reduced response for those with a fold change between the lower and the upper clinical cut-off $(=5.4)$, while a minimal response was predicted for isolates with a fold change above 5.4 .

\section{Statistical Analysis}

Treatment response was defined as a confirmed HIV-1 load $<50$ copies $/ \mathrm{ml}$ at week 24 . Analyses were performed on an intention-to-treat basis (all patients who started the TPV/r regimen were included).

Descriptive statistics were produced for demographic, clinical, virological and immunological characteristics of all cases and data are reported as median and interquartile range (IQR). Logistic regression was applied to analyze the association between virological response and clinical and virological variables. The variables included: age, gender, risk factor for the acquisition of HIV-1 infection, CDC staging, $\mathrm{HCV}$ and $\mathrm{HBV}$ co-infections, HIV-1 subtype, nadir of CD4 cell count, peak of HIV-RNA in the patient's history, prior virological suppression after HAART treatment, baseline CD4 count, baseline $\log _{10}$ HIV-RNA, number of previous antiretroviral regimens, previous exposure to antiretroviral drugs, previous treatment with enfuvirtide, number of active drugs in the new regimen according to Virtual Phenotype ${ }^{\mathrm{TM}}$-LM, total number of IAS PI-resistance associated mutations, predicted fold-change in $\mathrm{TPV} / \mathrm{r}$ susceptibility according to VP, TS, WS, PS, MS, and single protease point mutations.

Mutations detected in $>10 \%$ of patients with a $p<0.2$ value were retained for further analysis. A stepwise estimation model with backward procedure was used to select the set of mutations and clinical variables most strongly associated with virological response [12].

The prediction accuracy of each genotypic score was evaluated using Receiver Operating Characteristic (ROC); adjusted values for background drug activity (BAS, Background Activity Score: predicted viral load reduction by the OBR) ${ }^{3}$ were reported as well.

Data analysis was performed with STATA statistical package (release 9.0, 2006, Stata Corporation, College Station, Texas, USA).

\section{RESULTS}

\section{Patient Characteristics}

Baseline characteristics of patients enrolled in the study are shown in (Table 1). In addition to TPV/r, the new therapy included a NNRTI in $15 / 176$ patients $(8.5 \%)$, and enfuvirtide (with or without NNRTIs) in 99/176 patients $(56.2 \%), 71$ of whom were enfuvirtide-naïve and 28 were

\footnotetext{
${ }^{3}$ Hall DB, Baxter JD, Shapiro J, et al. Linear modeling to estimate the contribution of each drug component of the regimens of highly treatmentexperienced patients in RESIST. Presented at: at: XI European AIDS Conference Society; 24 - 27 October 2007; Madrid, Spain. Poster P4.3/71.
}

enfuvirtide-experienced. The median number of baseline IAS PI-resistance-associated mutations was 15 (IQR: 11-19); the median number of IAS TPV/r associated mutations was four (IQR: 2-5). The median and range values for each genotypic score in our population are also indicated. Fig. (1) presents the frequency of baseline protease mutations in our population. Median BAS was $0.75 \log (0.25-1)$.

\section{Treatment Efficacy and Predictors of Virological Response}

Overall, 139/176 patients completed the 24 week treatment follow-up. A total of $37 / 176$ patients (21\%) interrupted $\mathrm{TPV} / \mathrm{r}$ therapy for the following reasons: immuno-virological failure and/or clinical impairment $(36 \%)$, intolerance to $\mathrm{TPV} / \mathrm{r}(36 \%)$, and non-adherence (11\%). Four patients died during the follow-up, one of whom with an AIDS-related malignancy and the remaining three patients for non-AIDS-related events (lung cancer, drug overdose, and accidental death, respectively). A total of $61 / 159$ patients $(38.4 \%)$ at week 12 and 59/139 (42.4\%) patients at week 24, respectively, showed a pVL of $<50$ copies/ml. The median HIV-RNA $\log _{10}$ decrease was -2.0 (IQR $=-3$ to -0.3 ) after 12 weeks and -2.84 (IQR: -3.1 to $0.3)$ after 24 weeks. The median absolute CD4 variation was 60.5 (IQR: 10.5-137) cells at week 12 and 54 (IQR: 5-147) cells at week 24 .

\section{Univariate Analysis}

The variables significantly associated to outcome $(\mathrm{p} \leq$ 0.05 ) at univariate analysis are reported in Table 2.

CDC stage $C$ versus stage A (OR 2.25, 1.19-4.24 95\% $\mathrm{CI}, \mathrm{p}=0.013$ ) and the peak of HIV-RNA in the patient's history (OR 2.86, 1.55-5.27 95\% CI, $\mathrm{p}=0.001$ ) were associated with virological failure at week 24; while de novo use of enfuvirtide was correlated with virological success (OR 0.52, 0.27-0.97 95\% CI, $\mathrm{p}=0.042$ ). On the contrary, the number of active agents in the OBR was not a predictor of virological success in our cohort. The I13V and I54A/M/V substitutions among the IAS associated mutations, and N37DES, Q92K and I93LM among the non-IAS TPV/r associated mutations, resulted significantly associated with virological failure at week 24 by univariate analysis.

The Virtual Phenotype ${ }^{\text {TM }}$-LM TPV/r fold-change was significantly correlated with both the total number of protease mutations $(\mathrm{r}=0.44, \mathrm{p}<0.0001)$ and the IAS TPV $/ \mathrm{r}$ score $(\mathrm{r}=0.53, \mathrm{p}<0.0001)$. The predicted fold-change in $\mathrm{TPV} / \mathrm{r}$ susceptibility was also significantly associated to virological failure with univariate analysis (OR 1.53, 1.152.05 95\% CI, $\mathrm{p}=0.004)$.

All the TPV/r scores were associated with failure at week 24, including: TS (OR 1.35, 1.14-1.59 95\% CI, $\mathrm{p}<0.001$ ); WS (OR 1.18, 1.09-1.28 95\% CI, p<0.001); PS (OR 1.25, 1.09-1.43 95\% CI, $\mathrm{p}=0.001$ ) and MS, which showed, however, a weaker association with the outcome (OR 1.37, $1.00-1.8995 \% \mathrm{CI}, \mathrm{p}=0.051)$ compared to the other interpretation systems.

The accuracy of the scores in predicting the response to $\mathrm{TPV} / \mathrm{r}$ based therapy is shown in Fig. (2); the highest AUC value among the scores was obtained by WS (68\%). The cutoff which showed the highest specificity and sensitivity to 
Table 1. Baseline Characteristics of 176 PI-Experienced HIV Infected Patients

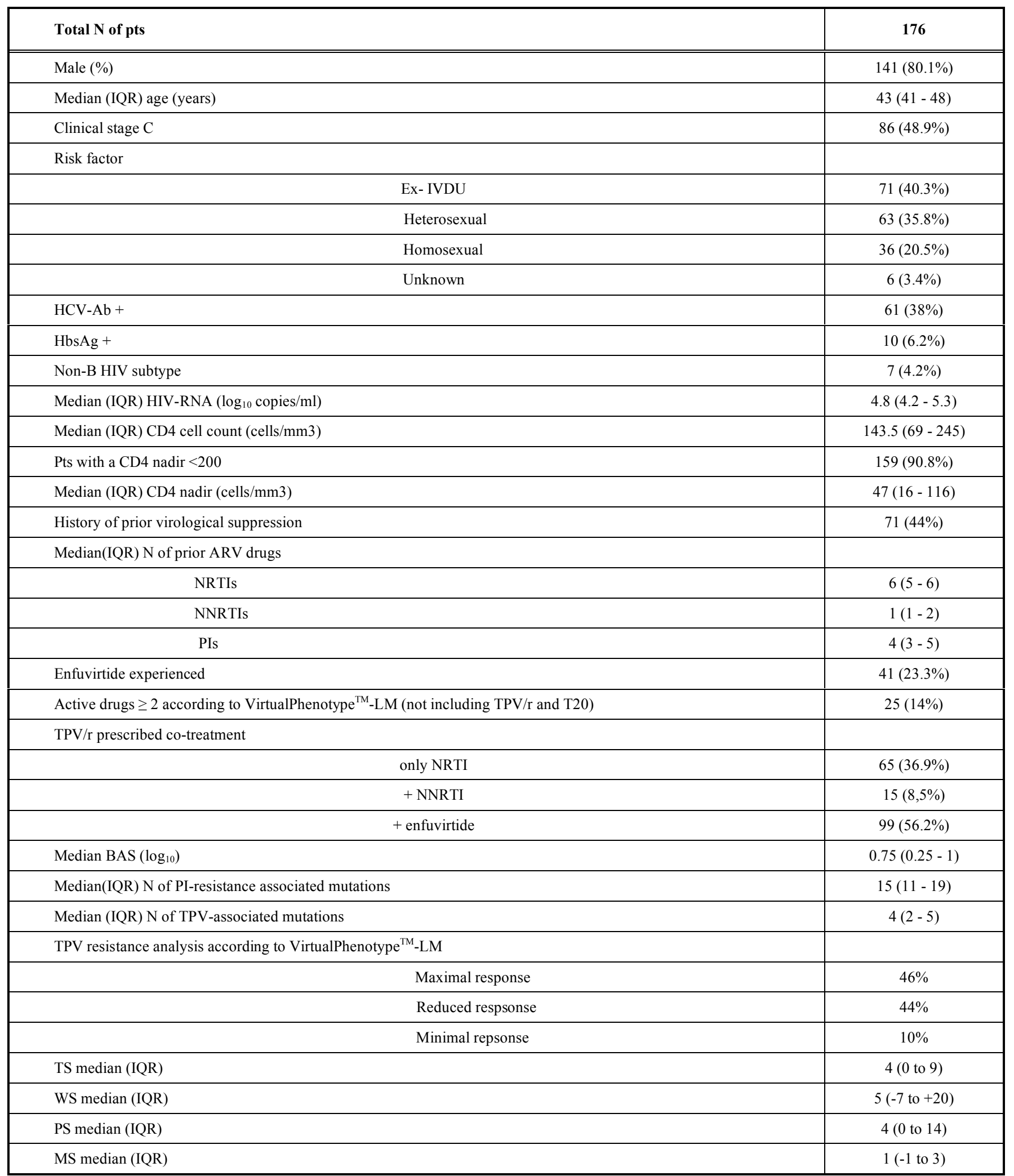

Legend: N: number; pts: patients; IQR: inter quartile range; IVDU: intravenous drug users; ARV: antiretroviral; NRTI: nucleoside retro transcriptase inhibitors; NNRTIs: nonnucleoside retro transcriptase inhibitors; PIs: protease inhibitors; TPV/r: tipranavir/ritonavir; BAS: Background Activity Score.

discriminate between virological responders/non-responders was 5 for WS and PS, 4 for TS, 1.5 for VP and 1 for MS.

Using the proposed cut-offs for $\mathrm{WS}^{2}$ ( $\leq 3=$ susceptible; $>3$ - $\leq 10=$ partially susceptible, and $\geq 10=$ resistant $)$, a virological response was achieved by $52 \%$ of susceptible patients $(n=38 / 73), 29 \%$ of partially susceptible $(n=22 / 75)$ and by $12 \%$ of subject classified as resistant $(n=3 / 25)$. 


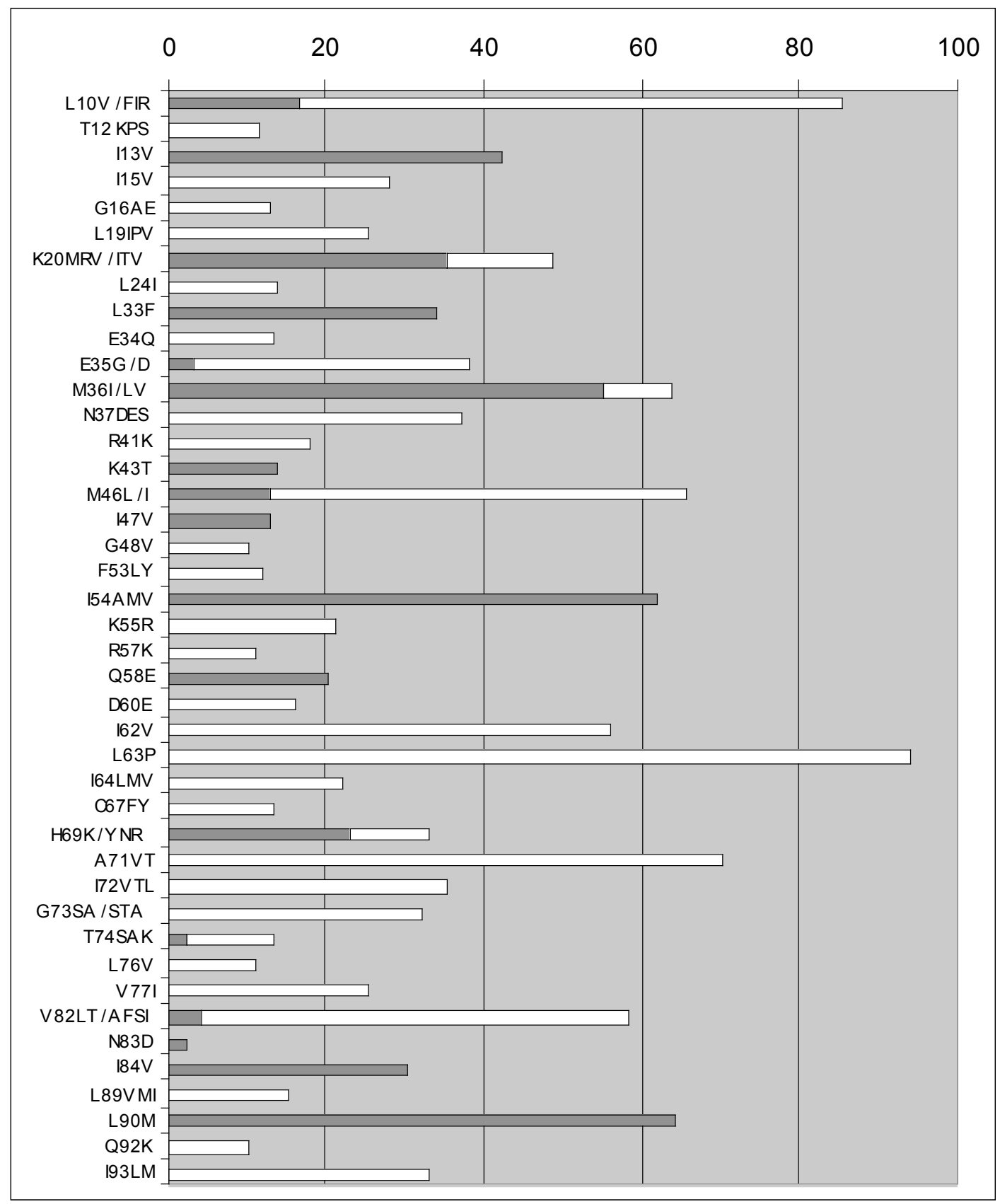

Fig. (1). Prevalence of baseline protease mutations in a population of 176 PI-experienced HIV patients. Legend: All mutations included in the IAS TPV-score (grey bars) and all other mutations at any position of the protease gene (white bars) showing a prevalence of amino acid substitutions with respect to wild type higher than $10 \%$ are reported.

The ROC curve adjusted for BAS showed the following AUC values for each score: TS $67.6 \%(95 \%$ CI 59.7-75.5), VP 61.8 (95\%CI 53-70.8), WS 69.8 (95\%CI 61.3-78.4), PS $65.7 \%$ (95\%CI 57.6-73.8), MS 58.5 (95\%CI 49.8-67.3).

\section{Multivariate Analysis}

A stepwise estimation model with backward procedure was then used to select the set of mutations most strongly associated with virological response. The mutations detected in $>10 \%$ of patients and showing a $\mathrm{p}$ value $<0.2$ with univariate analysis were entered in the analysis, including: L10V, T12KPS, I13V, I15V, G16AE, L19IPV, K20MR, L24I, L33F, E34Q, E35D, M36I, N37DES, R41K, K43T,
M46L, I47V, G48V, F53LY, I54AMV, K55R, Q58E, D60E, I62V, L63P, I64LMV, C67FY, H69K, A71VTIL, G73ACST, T74AKS, L76V, V77I, V82AFSI, I84V, L89IMV, L90M, I93LM. The following mutations were selected by the model as significantly $(\mathrm{p}<0.05)$ associated to failure: L33F (OR 2.86, 1.14-7.22, $\mathrm{p}=0.026$ ), I54AMV (OR $5.67,1.94-16.58, \mathrm{p}=0.002$ ), Q58E (OR 3.23, 1.06-9.90, $\mathrm{p}=0.039$ ), among IAS mutations, and N37DES (OR 2.30, $1.02-5.20, p=0.046)$ among non IAS mutations. On the contrary, V82AFSI (OR 0.20, 0.06-0.66, $\mathrm{p}=0.007$ ) and F53LY (OR 0.24, 0.07-0.78, $\mathrm{p}=0.018$ ) among non IAS mutations were associated with virological success (Table 2). 
Table 2. Univariate and Multivariate Analysis: Variables Associated with Virological Failure at Week 24

\begin{tabular}{|c|c|c|c|}
\hline & \multicolumn{3}{|c|}{ Univariate Analysis } \\
\hline & OR & $95 \%$ CI & P Value \\
\hline CDC stage_C & 2.25 & $1.19-4.24$ & 0.013 \\
\hline Peak of HIV-RNA in the patient's history & 2.86 & $1.55-5.27$ & 0.001 \\
\hline Use of T20 in T20-naïve pts & 0.52 & $0.27-0.97$ & 0.042 \\
\hline vPt TPV/r fold-change & 1.54 & $1.15-2.05$ & 0.004 \\
\hline Tot $N$ of mutations & 1.09 & $1.03-1.16$ & 0.003 \\
\hline TS (original TPV score) & 1.35 & $1.14-1.60$ & $<0.001$ \\
\hline WS (weighted TPV score) & 1.18 & $1.09-1.28$ & $<0.001$ \\
\hline PS (Parkin score) & 1.25 & $1.09-1.43$ & 0.001 \\
\hline MS (Marcelin score) & 1.37 & $1.00-1.89$ & 0.051 \\
\hline \multicolumn{4}{|l|}{ IAS TPV/r associated mutations } \\
\hline I13V & 2.08 & $1.10-3.94$ & 0.025 \\
\hline $\mathbf{I 5 4 A} / \mathrm{M} / \mathrm{V}$ & 2.42 & $1.24-4.71$ & 0.009 \\
\hline \multicolumn{4}{|l|}{ Other protease mutations } \\
\hline N37DES & 2.19 & $1.11-4.33$ & 0.024 \\
\hline Q92K & 4.81 & $1.06-21.80$ & 0.041 \\
\hline \multirow[t]{3}{*}{ I93LM } & 2.25 & $1.11-4.55$ & 0.025 \\
\hline & \multicolumn{3}{|c|}{ MULTIVARIATE ANALYSIS } \\
\hline & OR & $95 \%$ CI & P Value \\
\hline Use of T20 in T20-naïve pts & 0.44 & $0.20-0.94$ & 0.034 \\
\hline Peak of HIV-RNA in the patient's history & 2.71 & $1.40-5.24$ & 0.003 \\
\hline WS & 1.15 & $1.05-1.26$ & 0.004 \\
\hline \multicolumn{4}{|l|}{ IAS TPV/r associated mutations } \\
\hline L33F & 2.86 & $1.14-7.22$ & 0.026 \\
\hline I54AMV & 5.67 & $1.94-16.58$ & 0.002 \\
\hline Q58E & 3.23 & $1.06-9.90$ & 0.039 \\
\hline \multicolumn{4}{|l|}{ Other protease mutations } \\
\hline N37DES & 2.30 & $1.02-5.20$ & 0.046 \\
\hline V82AFSI & 0.20 & $0.06-0.66$ & 0.007 \\
\hline F53LY & 0.24 & $0.07-0.78$ & 0.018 \\
\hline
\end{tabular}

A p value $<0.05$ is considered significant (in bold).

A stepwise estimation model with backward procedure was also applied to all baseline clinical characteristics significantly associated to outcome with univariate analysis (Table 2) including WS, which showed the best AUC by ROC analysis. The use of T20 in T20 naïve patients (OR $0.44,0.20-0.94, p=0.034$ ) was independently associated with virological success, while the log peak of HIV-RNA (OR $2.71,1.40-5.24, \mathrm{p}=0.003)$ and WS (OR 1.15, 1.05-1.26, $\mathrm{p}=$ 0.004 ) resulted significantly associated with failure (Table 2 ).

\section{DISCUSSION}

Tipranavir is a non-peptidic protease inhibitor which has been proved to be active against some PI-resistant HIV-1 strains in vitro and could be included in salvage regimens for selected multi-experienced HIV patients. Understanding the role of predictors in virological response to $\mathrm{TPV} / \mathrm{r}$ can help clinicians to correctly identify patients who might benefit from this drug. With this aim, the relationship between baseline genotypic resistance and virological response to boosted tipranavir was evaluated in a group of PIexperienced patients.

When considering the resistance profile to $\mathrm{TPV} / \mathrm{r}$, as is well-known, a total of 21 mutations at 16 positions have been identified [9] and are currently listed in the IAS tables as TPV/r-associated [10], some of which are not involved in resistance to other PIs. Other genotypic scores, however, 


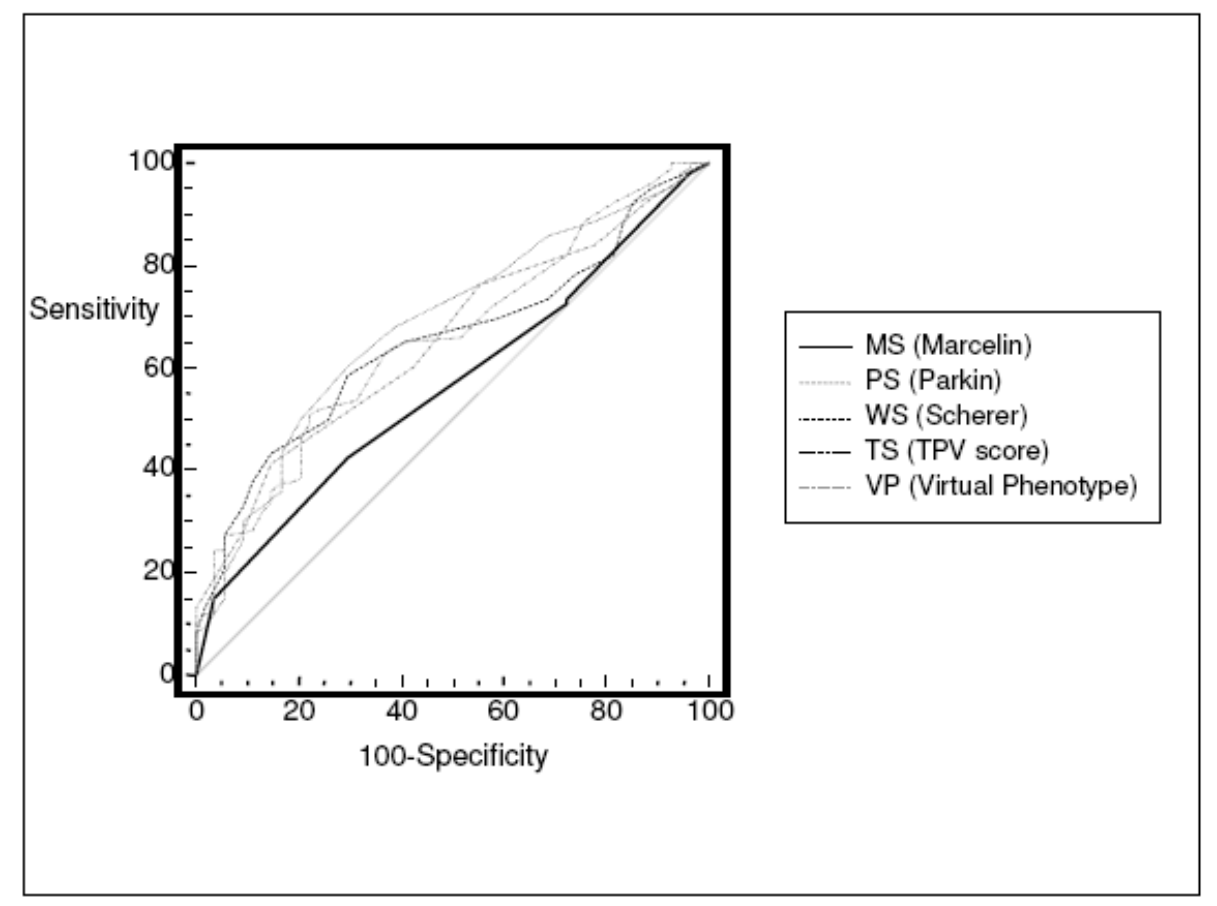

\begin{tabular}{|c|c|c|c|c|c|c|c|c|}
\hline & AUC & SE & $95 \% \mathrm{Cl}$ & Cut-off & Sensitivity & $95 \% \mathrm{Cl}$ & Specificity & $95 \% \mathrm{Cl}$ \\
\hline TS & 0.653 & 0.044 & 0.574 to 0.726 & $>4^{*}$ & 40.91 & $31.6-50.7$ & 85.71 & $74.6-93.2$ \\
\hline VP & 0.658 & 0.044 & 0.579 to 0.731 & $>15^{*}$ & 50.94 & $41.0-60.8$ & 77.78 & $64.4-87.9$ \\
\hline WS & 0.682 & 0.043 & 0.604 to 0.753 & $>5^{*}$ & 50.00 & $40.3-59.7$ & 79.37 & $67.3-88.5$ \\
\hline PS & 0.650 & 0.044 & 0.571 to 0.724 & $>5^{*}$ & 43.64 & $34.2-53.4$ & 84.13 & $72.7-92.1$ \\
\hline MS & 0.566 & 0.047 & 0.485 to 0.644 & $>1^{*}$ & 41.82 & $32.5-51.6$ & 74.60 & $62.1-84.7$ \\
\hline
\end{tabular}

Fig. (2). ROC curve analysis for the determination of clinical cut-offs. Legend: ROC curve synthetically describes the relationship between sensitivity and $100 \%$-specificity: the test is considered more valid when the area under the curve (AUC) approximates $100 \%$; on the contrary, the closer the AUC is to $50 \%$, the less valid is the result. The peak of the curve identifies the optimal cut-off to maximize sensitivity and specificity. This value corresponded to a cut-off $=5$ for WS which showed the largest AUC (68\%) from among the TPV/r genotyping scores analyzed.

have been proposed in the last year ${ }^{1}$ [11] and more recently, based on the data from the RESIST trials, a TPV/r-weighted score to predict virological response has been developed, in which only some mutations (I47V, I54AVM, Q58E, T74P, V82LT, and N83D) had greater weights in influencing virological failure, while other mutations (L24I, I50LV, I54L, L76V), not listed by IAS, were shown to confer an increased susceptibility to $\mathrm{TPV} / \mathrm{r}{ }^{2}$. Therefore, the applicability of four different TPV/r scores of baseline genotypic resistance (TS, WS, PS and MS) for predicting virological response was evaluated in our population. By univariate analysis, all these scores showed a good association with outcome. However, none could identify an easily applicable cut-off for the complex clinical management of our antiretroviral heavily experienced patients. When a ROC analysis was performed, in fact, none of these scores approached a sufficiently high AUC, even when adjusted for BAS, thus showing a reduced accuracy for predicting virological failures to TPV/r. Among these tests, the WS score proposed by Scherer et al. ${ }^{2}$ demonstrated the best performance with an AUC of $68 \%$ based on a cut-off of 5 mutations; the independent association of WS with virological failure was also confirmed by the multivariate analysis. However, a cut-off of 5 mutations is probably too low for this population in which a median number of four $\mathrm{TPV} / \mathrm{r}$-associated and $15 \mathrm{PI}$ - associated mutations was detected at baseline. Moreover, when patients were classified according to the proposed cut-offs for WS as susceptible (WS $\leq 3)$, partially susceptible $(>3-\leq 10)$ and resistant $(\geq$ $10)$, while WS could efficiently individuate patients who would not respond to a TPV/r based regimen (in fact, $88 \%$ of patient classified as resistant with a WS $>10$ did not respond to the new therapy in our study), only one half of patients classified as susceptible demonstrated a virological response. Therefore, additional variables, not considered by genotypic scores, such as adherence or toxicities and pharmacokinetics aspects, supposedly influence virological success independently of baseline genotypes. These results are undoubtedly limited by the sample size; however, they also highlight the difficulty of predicting response with mutational scores which vary considerably with the patient populations and statistical methods used to derive them.

Furthermore, the TPV/r susceptibility fold-change, as predicted by the Virtual Phenotype ${ }^{\mathrm{TM}}-\mathrm{LM}$, demonstrated a good correlation with the baseline number of protease and $\mathrm{TPV} / \mathrm{r}$-associated mutations in our population and was also associated with failure with univariate analysis. Actually, we arbitrarily used the Virtual Phenotype ${ }^{\mathrm{TM}}-\mathrm{LM}$, which accurately predicts the real phenotype [13], to quantify the $\mathrm{TPV} / \mathrm{r}$ resistance levels, even if we are aware that the 
accuracy of this approach may be lower for newly-approved drugs.

Differences among scores were also evidenced by the analysis of the association of single mutated positions in the protease gene with the virological outcome. Only three mutations (L33F, I54AMV, Q58E) among those included in the IAS list were associated with virological failure with multivariate analysis. The role of mutations I54AMVand $\mathrm{Q} 58 \mathrm{E}$ in determining resistance to $\mathrm{TPV} / \mathrm{r}$ is recognized by all the proposed genotypic scores. In particular, WS attributes one of the highest scores $(=5)$ to mutation Q58E, which is also the only IAS TPV/r-associated mutation retained in the MS score attributing it a score of one, as in TS and in PS. It is remarkable that $\mathrm{Q} 58 \mathrm{E}$ is one of the "novel" protease mutations not previously involved in resistance to other PIs. Parkin et al. [14], included the Q58E mutation among those associated with lopinavir resistance; in addition, this mutation is detected more frequently in patients who have been treated with more than three PIs, and clusters with mutations at positions $10,46,54$ and 82 [15] which are all included in the original TPV/r score. On the contrary, the role of mutation $\mathrm{L} 33 \mathrm{~F}$ is more controversial; only in PS was this mutation linked to virological falure, while WS considers it as a predictor of virological success; it was however, one of the four mutations initially associated to $\mathrm{TPV} / \mathrm{r}$ resistance and its importance is probably underestimated by the WS and MS scores. The potential role of other protease mutations, not included in the IAS list, on virological response to $\mathrm{TPV} / \mathrm{r}$ was also evaluated in our population; in this setting, mutation N37DES resulted associated with failure, while mutations F53LY and V82AFS, which have been linked to resistance to other drugs such as lopinavir/rtv, were identified by the stepwise backward analysis as associated with virological success. Interestingly, these results confirm the finding of Marcelin et al. [11] regarding the beneficial effect of mutation L53LY on virological response to TPV/r.

In our multi-experienced population, high levels of virological response to a new regimen based on $\mathrm{TPV} / \mathrm{r}$ (reaching a pVL $>1 \log$ decrease in $62 \%$ of cases and $<50$ copies $/ \mathrm{ml}$ in $42 \%$ of cases after 24 weeks, respectively) were obtained. While clinical variables such as the patient peak viral load and a more advanced stage of HIV related disease were associated with failure, the inclusion in the combined regimen of an agent from a new antiretroviral class, such as enfuvirtide, was definitely associated to a better virological outcome, as previously demonstrated by the phase II and III registration trials [2-4], and probably masked the influence of the number of OBR active agents on the virological response.

In conclusion, this study suggests that the "weighted" approach, which attributes a different value to single aminoacid substitutions in affecting the therapy response, is more qualified than unweighted scores to interpret correctly the antiretroviral resistance profile of multi-experienced patients, even if it was not possible to determine a cut-off which could be easily applied for discriminating responders. This difficulty also further underlines the importance of validating genotypic scores outside of phase II/III clinical trials in order to verify their performance in clinical settings.

\section{ACKNOWLEDGEMENTS}

Participants in the Italian EAP BI 1182.16:

G. Angarano, A. Saracino, A. Tartaglia, D. Cibelli (Foggia); F. Suter, F. Maggiolo, D. Ripamonti, M. Airoldi (Bergamo); G. Di Perri, S. Bonora (Torino); M. Galli, S. Rusconi (Milano); A. Cargnel, V. Micheli (Milano); F. Mazzotta, S. Lo Caputo, P. Pierotti (Firenze); P. Grossi, L. Lazzaroni (Varese); B. Grisorio, S. Ferrara (Foggia); G. Pastore, L. Monno, N. Ladisa, P. Maggi (Bari); G. Parruti (Pescara); P. Narciso, R. Bellagamba (Roma); C.F. Perno, F. Forbici (Roma); G. Carosi, P. Nasta (Brescia); C. Tinelli (Pavia).

We also thank Ms. Paulene Butts for the review of the manuscript and Gianfranco Botte for secretarial assistance.

\section{FUNDING}

The study was partly supported by an unrestricted grant of Boehringer Ingelheim - Italy.

$\begin{array}{ll}\text { ABBREVIATIONS } \\ \text { IAS } & =\text { International AIDS Society } \\ \text { HAART } & =\text { Highly Active Antiretroviral Therapy } \\ \text { PI } & =\text { Protease Inhibitors } \\ \text { TPV/r } & =\text { Tipranavir/ritonavir } \\ \text { FC } & =\text { Fold-Change } \\ \text { TS } & =\text { Tipranavir Score } \\ \text { PS } & =\text { Parkin Score } \\ \text { MS } & =\text { Marcelin Score } \\ \text { WS } & =\text { Weighted Score } \\ \text { EAP } & =\text { Early Access Program } \\ \text { AST } & =\text { Aspartate Aminotransferase } \\ \text { ALT } & =\text { Alanine Aminotransferase } \\ \text { T20 } & =\text { Enfuvirtide } \\ \text { VP } & =\text { Virtual Phenotype }{ }^{\text {TM }} \text {-LM, } \\ \text { IQR } & =\text { Interquartile Range } \\ \text { ROC } & =\text { Receiver Operating Characteristic } \\ \text { AUC } & =\text { Area Under Curve }\end{array}$

\section{REFERENCES}

[1] Zaccarelli M, Tozzi V, Lorenzini P, et al. Collaborative Group for Clinical Use of HIV Genotype Resistance Test (GRT) at National Institute for Infectious Diseases Lazzaro Spallanzani. Multiple drug class-wide resistance associated with poorer survival after treatment failure in a cohort of HIV-infected patients. AIDS 2005; 19: 1081-9.

[2] Gathe J, Cooper DA, Farthing C, et al. Efficacy of the protease inhibitors tipranavir plus ritonavir in treatment-experienced patients: 24-week analysis from the RESIST-1 trial. Clin Infect Dis 2006; 43(10): 1337-46.

[3] Cahn P, Villacian J, Lazzarin A, et al. Ritonavir-boosted tipranavir demonstrates superior efficacy to ritonavir-boosted protease inhibitors in treatment-experienced HIV-infected patients: 24-week results of the RESIST-2 trial. Clin Infect Dis 2006; 43: 1347-56.

[4] Hicks CB, Cahn P, Cooper DA, et al. Durable efficacy of tipranavir-ritonavir in combination with an optimised background regimen of antiretroviral drugs for treatment-experienced HIV-1infected patients at 48 weeks in the Randomized Evaluation of 
Strategic Intervention in multi-drug resistant patients with Tipranavir (RESIST) studies: an analysis of combined data from two randomised open-label trials. Lancet 2006; 368: 466-75.

[5] Katlama C, Esposito R, Gatell JM, et al. Efficacy and safety of TMC114/ritonavir in treatment-experienced HIV patients: 24-week results of POWER 1. AIDS 2007; 21: 395-402.

[6] Panel on Antiretroviral Guidelines for Adult and Adolescents. Guidelines for the use of antiretroviral agents in HIV-1-infected adults and adolescents. Department of Health and Human Services. November 3, 2008; 1-139. Available at http://www.aidsinfo.nih.gov/ContentFiles/AdultandAdolescentGL. pdf. Accessed (November 20, 2008).

[7] Rusconi S, La Seta Catamancio S, Citterio P, et al. Susceptibility to PNU-140690 (Tipranavir) of human immunodeficiency virus type 1 isolates derived from patients with multidrug resistance to other protease inhibitors. Antimicrob Agents Chemother 2000; 44: 132832.

[8] Doyon L, Tremblay S, Bourgon L, et al. Selection and characterization of HIV-1 showing reduced susceptibility to the non-peptidic protease inhibitor tipranavir. Antiviral Res 2005; 68: 27-35.

[9] Baxter JD, Schapiro JM, Boucher CA, et al. Genotypic changes in human immunodeficiency virus type 1 protease associated with reduced susceptibility and virologic response to the protease inhibitor tipranavir. J Virol 2006; 80: 10794-801.

[10] Johnson VA, Brun-Vezinet F, Clotet B, et al. Update of the drug resistance mutations in HIV-1: Spring 2008. Top HIV Med 2008; 16(1): 62-8.

[11] Marcelin AG, Masquelier B, Descamps D, et al. Tipranavirritonavir genotypic resistance score in protease inhibitorexperienced patients. Antimicrob Agents Chemother 2008 Sep; 52(9): 3237-43.

[12] Flandre P. Clinically validated genotype analysis. Antivir Ther 2004; 9: 1037-8.

[13] Perez-Elias MJ, Garcia-Arota I, Muñoz V, et. al.. Phenotype or virtual phenotype for choosing antiretroviral therapy after failure: a prospective, randomized study. Antivir Ther 2003 Dec; 8(6): 57784.

[14] Parkin NT, Chappey C, Petropoulos CJ. Improving lopinavir genotype algorithm through phenotype correlations: novel mutation patterns and amprenavir cross-resistance. AIDS 2003; 17: 955-61.

[15] Svicher V, Ceccherini-Silberstein F, Erba F, et al. Novel HIV-1 protease mutations potentially involved in resistance to protease inhibitors. Antimicrob Agents Chemother 2005; 49: 2015-25. 